\title{
ABOUT COMPATIBILITY OF THE VEKUA EQUATION WITH SOME OTHER EQUATIONS
}

\author{
Slagjana Brsakoska
}

\begin{abstract}
In the paper, the compatibility of the Vekua equation with equations that define some generalized analytical functions will be considered. The main results are formulated in a few theorems.
\end{abstract}

\section{INTRODUCTION}

The equation

$$
\frac{\hat{d} W}{d \bar{z}}=A W+B \bar{W}+F
$$

where $A=A(z), B=B(z)$ and $F=F(z)$ are given complex functions from a complex variable $z \in D \subseteq \mathbb{C}$ is the well known Vekua equation [1] according to the unknown function $W=W(z)=u+i v$. The derivative on the left side of this equation has been introduced by G. V. Kolosov in 1909 [2]. During his work on a problem from the theory of elasticity, he introduced the expressions

$$
\frac{1}{2}\left[\frac{\partial u}{\partial x}+\frac{\partial v}{\partial y}+i\left(\frac{\partial v}{\partial x}-\frac{\partial u}{\partial y}\right)\right]=\frac{\hat{d} W}{d z}
$$

and

$$
\frac{1}{2}\left[\frac{\partial u}{\partial x}-\frac{\partial v}{\partial y}+i\left(\frac{\partial v}{\partial x}+\frac{\partial u}{\partial y}\right)\right]=\frac{\hat{d} W}{d \bar{z}}
$$

known as operator derivatives of a complex function $W=W(z)=u(x, y)+i v(x, y)$ from a complex variable $z=x+i y$ and $\bar{z}=x-i y$ corresponding. The operating rules for this derivatives are completely given in the monograph of Г. Н. Положий [3] (page18-31). In the mentioned monograph are defined so cold operator integrals

$$
\hat{\int} f(z) d z \text { and } \hat{\int} f(z) d \bar{z}
$$

from $z=x+i y$ and $\bar{z}=x-i y$ corresponding (page 32-41). As for the complex integration in the same monograph is emphasized that it is assumed that all operator integrals can be solved in the area $D$.

2010 Mathematics Subject Classification. Primary: 34M45

Key words and phrases. Vekua equation, areolar derivatives, areolar equation, p-analytic functions, (p,q)-analytic functions 
In the Vekua equation (1) the unknown function $W=W(z)$ is under the sign of a complex conjugation which is equivalent to the fact that $B=B(z)$ is not identically equaled to zero in $D$. That is why for (1) the quadratures that we have for the equations where the unknown function $W=W(z)$ is not under the sign of a complex conjugation, stop existing.

This equation is important not only for the fact that it came from a practical problem, but also because depending on the coefficients $A, B$ and $F$ the equation (1) defines different classes of generalized analytic functions. For example, for $F=F(z) \equiv 0$ in $D$ the equation (1), i.e. $\frac{\hat{d} W}{d \bar{z}}=A W+B \bar{W}$ defines so cold generalized analytic functions from fourth class; and for $A \equiv 0$ and $F \equiv 0$ in $D$, the equation (1) i.e. the equation $\frac{\hat{d} W}{d \bar{z}}=B \bar{W}$ defines so cold generalized analytic functions from third class or the $(r+i s)$-analytic functions [3], [4].

Those are the cases when $B \neq 0$. But if we put $B \equiv 0$, we get the following special cases. In the case $A \equiv 0, B \equiv 0$ and $F \equiv 0$ in the working area $D \subseteq \mathbb{C}$ the equation (1) takes the following expression $\frac{\hat{d} W}{d \bar{z}}=0$ and this equation, in the class of the functions $W=u(x, y)+i v(x, y)$ whose real and imaginary parts have unbroken partial derivatives $u_{x}^{\prime}, u_{y}^{\prime}, v_{x}^{\prime}$ and $v_{y}^{\prime}$ in $D$, is a complex writing of the Cauchy-Riemann conditions. In other words it defines the analytic functions in the sense of the classic theory of the analytic functions. In the case $B \equiv 0$ in $D$, i.e. $\frac{\hat{d} W}{d \bar{z}}=A W+F$ is the so cold areolar linear differential equation [3] (page 39-40) and it can be solved with quadratures by the formula:

$$
W=e^{\hat{\int} A(z) d \bar{z}}\left[\Phi(z)+\hat{\int} F(z) e^{-\hat{\int} A(z) d \bar{z}} d \bar{z}\right] .
$$

here $\Phi=\Phi(z)$ is an arbitrary analytic function in the role of an integral constant.

\section{MAIN RESUlt}

We will consider the Vekua equation in canonical shape

$$
\frac{\hat{d} w}{d \bar{z}}=B \bar{w}+F
$$

and some theorems regarding this equation and equations that define the generalized analytic functions shall be proved. The idea is to find their common solutions.

\section{1. p-ANALYTIC FUNCTIONS THAT ARE SOLUTIONS OF THE CANONICAL VEKUA EQUATION}

According to the definition of the p-analytic functions in [3], they are defined as solutions of the complex differential equation of first order 


$$
\frac{\hat{d} w}{d \bar{z}}=P(z, \bar{z}) \frac{\hat{d} \bar{w}}{d \bar{z}}
$$

We ask the question which is the common solution of the equations (5) and (6) if it exists?

Let $W=W(z, \bar{z})$ is a p-analytic function which in the same time is a solution to the Vekua equation (5). That means that the function $W=W(z, \bar{z})$ satisfies the equations (5) and (6). These equations have equal left sides, so if we equal the right sides, too we get:

$$
P(z, \bar{z}) \frac{\hat{d} \bar{W}}{d \bar{z}}=B \bar{W}+F .
$$

If we divide with $P(z, \bar{z}) \neq 0$ in the last expression and we conjugate the whole equation, we get:

$$
\begin{aligned}
& \frac{\hat{d} \bar{W}}{d \bar{z}}=\frac{B}{P} \bar{W}+\frac{F}{P} \\
& \frac{\hat{d} \bar{W}}{d \bar{z}}=\frac{B}{P} \bar{W}+\frac{F}{P} \\
& \frac{\hat{d} W}{d z}=\frac{\bar{B}}{\bar{P}} W+\frac{\bar{F}}{\bar{P}}
\end{aligned}
$$

The last equation is a linear complex differential equation which can be solved explicitly and it's solution has the following equation

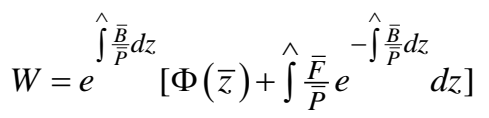

where $\Phi=\Phi(\bar{z})$ is an arbitrary analytic function in the role of an integral constant. Here we are using the property of the conjugation of an areolar derivative [3], i.e. $\frac{\bar{d} \bar{W}}{d \bar{z}}=\frac{\hat{d} W}{d z}$. So, we have proved the following theorem:

Theorem 1. The class of complex functions (7) are common solutions of the equations (5) and (6).

\section{2. (p,q)-analytic functions that are solutions of the canonical Vekua equation}

According to the definition of the (p,q)-analytic functions in [3], they are defined as solutions of the complex differential equation of first order

$$
P(z, \bar{z}) \frac{\hat{d} w}{d \bar{z}}+Q(z, \bar{z}) \frac{\hat{d} \bar{w}}{d \bar{z}}=0,
$$

where

$$
\begin{aligned}
& P(z, \bar{z})=p\left(\frac{z+\bar{z}}{2}, \frac{z-\bar{z}}{2 i}\right)+1-i q\left(\frac{z+\bar{z}}{2}, \frac{z-\bar{z}}{2 i}\right), \\
& Q(z, \bar{z})=p\left(\frac{z+\bar{z}}{2}, \frac{z-\bar{z}}{2 i}\right)-1-i q\left(\frac{z+\bar{z}}{2}, \frac{z-\bar{z}}{2 i}\right) .
\end{aligned}
$$

We ask the question which is the common solution of the equations (5) and (8) if it exists? 
Let $W=W(z, \bar{z})$ is a $(\mathrm{p}, \mathrm{q})$-analytic function which in the same time is a solution to the Vekua equation (5). That means that the function $W=W(z, \bar{z})$ satisfies the equations (5) and (8). If we put the equation (8) in the following form

$$
\frac{\hat{d} W}{d \bar{z}}=-\frac{Q(z, \bar{z})}{P(z, \bar{z})} \cdot \frac{\hat{d} \bar{W}}{d \bar{z}}
$$

then the equations (5) and (9) have equal left sides, so if we equal the right sides, too we get:

$$
-\frac{Q(z, \bar{z})}{P(z, \bar{z})} \cdot \frac{\hat{d} \bar{W}}{d \bar{z}}=B \bar{W}+F .
$$

If we divide with $-\frac{Q(z, \bar{z})}{P(z, \bar{z})}$ in the last expression and we conjugate the whole equation, we get:

$$
\begin{aligned}
& \frac{\hat{d} \bar{W}}{d \bar{z}}=-\frac{P B}{Q} \bar{W}-\frac{P F}{Q} \\
& \frac{\hat{d} \bar{W}}{d \bar{z}}=-\frac{P B}{Q} \bar{W}-\frac{P F}{Q} \\
& \frac{\hat{d} W}{d z}=-\frac{\bar{P} \bar{B}}{\bar{Q}} W-\frac{\bar{P} \bar{F}}{\bar{Q}}
\end{aligned}
$$

The last equation is a linear complex differential equation which can be solved explicitly and it`s solution has the following equation

$$
W=e^{-\hat{\int} \frac{\bar{P} \bar{B}}{\bar{Q}} d z}\left[\Phi(\bar{z})-\hat{\int} \frac{\bar{P} \bar{F}}{\bar{Q}} e^{\hat{\int} \frac{\bar{P} \bar{B}}{\bar{Q}} d z} d z\right]
$$

where $\Phi=\Phi(\bar{z})$ is an arbitrary analytic function in the role of an integral constant. Here we are using the same above mentioned property of the conjugation of an areolar derivative. So, we have proved the following theorem:

Theorem 2. The class of complex functions (10) are common solutions of the equations (5) and (8).

Remark. The solutions (7) and (10) define enough large class of functions which has the property to be the solutions of the complex canonical differential Vekua equation.

\section{References}

[1] Н. И. Векуа, Обобщение аналитические функици, Наука, Москва, 1988.

[2] Г. В. Колосов, Об одном приложении теории функиий комплесного переменного к плосной задаче математической теории упругости, Юрьев, 1909. 
[3] Г. Н. Положий, Обобщение теории аналитических функиий комплесного переменного р-аналитические и (p,q)-аналитические функиий и некоторае их применения, Киев. 1965.

[4] S. Brsakoska, Operator differential equations from the aspect of the generalized analytic functions, MSc thesis, Skopje, 2006.

Ss. Cyril and Methodius University, Faculty of Natural Sciences and Mathematics, Institute of Mathematics, Arhimedova St. 5,Skopje, Republic of Macedonia e-mail: sbrsakoska@gmail.com 